\title{
MicroRNA and target mRNA selection through invasion and cytotoxicity cell modeling and bioinformatics approaches in esophageal squamous cell carcinoma
}

\author{
MU LU ${ }^{1 *}$, YAQIN SONG ${ }^{2 *}$, WENBO FU ${ }^{3}$, YANG LIU $^{1}$, SHITAO HUAI $^{1}$, \\ XIAOBIN CUI ${ }^{4}$, LIJUAN PANG $^{4}, \mathrm{LAN} \mathrm{YANG}^{4}$ and YUTAO WEI ${ }^{5}$ \\ ${ }^{1}$ Medical College of Shihezi University, Shihezi, Xinjiang; ${ }^{2}$ Department of Nutrition, Jining No. 1 People's Hospital, \\ Jining, Shandong; ${ }^{3}$ Department of Cardiology, People's Hospital of Xinjiang Uygur Autonomous Region, Urumqi; \\ ${ }^{4}$ Department of Pathology, Medical College of Shihezi University; ${ }^{5}$ Department of Thoracic and Cardiovascular Surgery, \\ First Hospital Affiliated to Medical College of Shihezi University, Shihezi, Xinjiang, P.R. China
}

Received January 16, 2017; Accepted June 9, 2017

DOI: $10.3892 / o r .2017 .5776$

\begin{abstract}
This study analyzed microRNA (miRNA) and mRNA expression profiles and investigated the biological characteristics of ESCC by using invasion and cytotoxicity cell models. miRNA profiles were evaluated through miRNA microarray. Transwell chamber and nedaplatin (NDP) were used to construct invasion and cytotoxicity cell models. Invasion Transwell and cytotoxicity assays were performed to examine the invasiveness and proliferation in the cell models. Functional miRNAs were selected from dysregulated miRNAs through qRT-PCR. Biometric Research Program (BRB)-array tools, Cytoscape plugins, and DAVID were utilized to find potential mRNAs targeted by these two miRNAs between ESCC and paired normal adjacent tissues. Our microarray obtained 11 dysregulated miRNAs expressed in three paired ESCC samples from Kazakhs (ethnicity in Northwestern China). qRT-PCR demonstrated the miRNA expression in the invasion and cytotoxicity cell models. miR-652-5p and miR-21-5p exhibited a consistent expression level in the microarray and cell models. Bioinformatics revealed that the potential targets of PLD1, MSH2, STC1, and DSG1 might be involved in ESCC invasion and proliferation. Cell models with
\end{abstract}

Correspondence to: Dr Yutao Wei, Department of Thoracic and Cardiovascular Surgery, First Hospital Affiliated to Medical College of Shihezi University, 107 North No. 2 Road, Shihezi, Xinjiang 832000, P.R. China

E-mail:wytfwb@126.com

Dr Lan Yang, Department of Pathology, Medical College of Shihezi University, Shihezi, Xinjiang, P.R. China

E-mail: 417428883@qq.com

*Contributed equally

Key words: miRNA, cell model, microarray, invasion, cytotoxicity, bioinformatics bioinformatics approaches may help distinguish functional genes. miR-652-5p, miR-21-5p, and their potential target genes may participate in ESCC development and metastasis.

\section{Introduction}

Esophageal carcinoma (EC) is a common malignancy that affects $>450,000$ individuals worldwide and causes high morbidity and mortality. Esophageal squamous cell carcinoma (ESCC) and esophageal adenocarcinoma are the two major histologic types of EC (1). ESCC is a frequent type of histopathology in China, especially in Xinjiang and Northwestern regions, where ESCC in Kazakhs is abnormally higher than that in other minorities (2). Although treatment methods have improved, ESCC has become a major public health concern because of its increasing mortality. Therefore, we should further explore the molecular mechanisms related to the pathogenesis of EC to develop effective treatments.

MicroRNAs (miRNAs) are a class of small (21-25 nucleotides) naturally occurring non-coding RNAs. miRNAs can perform dual functions as a tumor promotor or suppressor by targeting different mRNAs or dysregulating their expression levels. Their regulatory mechanisms occur through the posttranscriptional repression of their target genes by targeting 3'UTR (3). miRNAs are highly and extensively regulated, and miRNA binding sites can be present in 5'UTR and within coding sequences (4). miRNAs are highly conserved in species (5). With tissue specificity, miRNAs are suitable for early cancer detection and prognosis prediction (6). Mature miRNAs play important regulatory roles in cell proliferation, differentiation, and death (7). Therefore, miRNAs can be used as biomarkers to diagnose and treat diseases, including ESCC, whose early recognition is crucial. The role of miRNAs in the pathological process of ESCC has also been widely examined (8). For instance, Chen et al (9) used microarrays to examine the miRNA expression profiles of 119 paired ESCC tissue samples and indicated a four-miRNA signature that potentially predicts patient survival. Takeshita et al performed a miRNA array by using serum samples from ESCC patients 
and found that miR-1246 can be strongly utilized as a novel diagnostic and prognostic ESCC biomarker (10). The role of miRNAs has also been demonstrated in the diagnostic and prognostic uses of circulation in ESCC patients.

Metastasis and proliferation are related to cancer prognosis, and various steps of metastasis involve several miRNAs (11). miRNA arrays have been used to determine the functions of dysregulated miRNAs in cancer. However, we have yet to reveal the mechanisms on how to effectively screen key miRNAs in dysregulated genes. First, we aimed to examine the miRNA microarray platforms in ESCC tissue samples and evaluate the miRNA expression levels by using an ESCC cell line subpopulation with high invasive ability and an ESCC cell line treated with the anticancer drug nedaplatin (NDP). We also performed qRT-PCR to explore the miRNAs involved in cell invasion and proliferation. Second, we applied a systems bioinformatics approach to investigate the biological characteristics of dysregulated miRNAs in ESCC and to elucidate their molecular mechanisms in tumor development and invasion. Finally, we selected relevant functional miRNAs.

\section{Materials and methods}

Patients and specimens. ESCC tissues and related non-tumor normal esophageal tissues were obtained from 8 ChineseKazakh ESCC patients undergoing esophagectomy in 2015 at The First Affiliated Hospital of the Medical College, Shihezi University. None of these patients received pre-surgical radiochemotherapy. The excised specimens were quickly frozen and stored in liquid nitrogen until RNA was extracted. A pathologist in The First Affiliated Hospital of the Medical College, Shihezi University provided distinct histological confirmation of the ESCC diagnosis. The clinicopathological information of all of the patients is shown in Table I. This study was approved by the ethics committees of The First Affiliated Hospital of the Medical College, Shihezi University, and informed consent was obtained from each patient.

Agilent miRNA array and computational analysis. ESCC tissues were transported to Shanghai Biotechonology Co., Ltd. (Shanghai, China) for miRNA isolation and microarray assays were performed by using an Agilent Human miRNA microarray platform (design ID: 38169). Labeling and hybridization were performed using the protocols. Only the first three pairs of ESCC tissues (Table I) could be used for further processes. The slides were scanned using an Agilent microarray scanner (Agilent Technologies, CA, USA) and Feature Extraction 10.7. Raw data were normalized using the quantile algorithm of Gene Spring Software 12.6 (Agilent Technologies). Student's t-test was conducted to screen differentially expressed genes. The filtration criteria were as follows: i) fold change (linear) $\leq 0.5$ or fold change (linear) $\geq 2$ and ii) t-test $\mathrm{P}<0.05$.

Cell lines and cell culture. ECA109 is a human ESCC cell line obtained from Shihezi University (Xinjiang, China). The cell line was cultured in Roswell Park Memorial Institute (RPMI)-1640 nutrient mixture (Life Technologies, Grand Island, NY, USA) supplemented with $10 \%$ fetal bovine serum (FBS) in a humidified atmosphere containing $5 \% \mathrm{CO}_{2}$ at $37^{\circ} \mathrm{C}$.
Construction of highly invading cell models. Highly invading cell models were selected in accordance with a previously described method (12). Subpopulations from the ECA109 ESCC cell line were chosen by using a Corning Matrigel $^{\mathrm{TM}}$ invasion chamber (NY, USA) with a 6 -well plate and $8.0-\mu \mathrm{m}$ pores. ECA109 $\left(2 \times 10^{4}\right.$ cells) was seeded into the wells and suspended in $600 \mu \mathrm{l}$ of RPMI-1640 containing 10\% FBS. After the cells were cultured at $37^{\circ} \mathrm{C}$ for $48 \mathrm{~h}$, the chambers were removed. The invasion ability of the cells that invaded the lower chamber was higher than that of the cells in other parts of the chamber. After an appropriate number of cells was set, the next round of selection was conducted. Among the first-round selections, the sublines in the lower chambers were nominated as ECA109T1-1, the sublines from the second and third rounds were, respectively, designated as ECA109T1-2 and ECA109T1-3. The parental line in the first series was designated ECA109T1. If subpopulations were passaged $>20$ times, a new round of selection was performed.

Invasion Transwell assays. The cells were starved with an Opti-MEM (Corning, NY, USA) medium for $6 \mathrm{~h}$. Then, $10^{6}$ cells were suspended in $1 \mathrm{ml}$ of serum-free medium, and $100 \mu 1$ or $10 \times 10^{4}$ cells were plated onto the upper chamber of a 24-well plate with $8.0-\mu \mathrm{m}$ pores Corning Matrigel Invasion Chamber and coated with $0.7 \mathrm{mg} / \mathrm{ml}$ Matrigel (BD Biosciences. They were placed in $600 \mu \mathrm{l}$ of RPMI-1640 medium containing $10 \%$ FBS in the lower chamber. The cells were incubated for $48 \mathrm{~h}$, and those on the lower surface of the filter were fixed using paraformaldehyde and stained with $0.5 \%$ crystal violet. Five fields across the lower membrane were photographed at x100 magnification. The cells were then counted using ImageJ (http://rsbweb.nih.gov/ij/).

Construction of cytotoxicity cell model and assay. ECA109 was seeded into a 96-well microplate at a population of 4,000 cells per well and cultured for $12 \mathrm{~h}$ in RPMI-1640 medium with $10 \%$ FBS. Then, the cells were treated with $10 \mu \mathrm{l}$ of $64 \mathrm{mg} / \mathrm{l} \mathrm{NDP}$ in the Opti-MEM (Corning) medium. The NDP concentration was compared with the results of $\mathrm{Su}$ et al (13). The cells incubated in the Opti-MEM medium were set up with the control group and incubated for $48 \mathrm{~h}$ at $37^{\circ} \mathrm{C}$. Afterwards, each well was mixed with $10 \mu \mathrm{l}$ of CCK-8 (Seabiotech, Shanghai, China) and further incubated for $3 \mathrm{~h}$ at $37^{\circ} \mathrm{C}$. Optical density (OD) was detected by using a spectrophotometer (Bio-Rad, Hercules, CA, USA) at $450 \mathrm{~nm}$. Growth inhibition was calculated as the percentage of the untreated controls. To construct the cytotoxicity cell model, we treated the ECA109 cells with $200 \mu \mathrm{l}$ of NDP $(64 \mathrm{mg} / \mathrm{l})$ for $48 \mathrm{~h}$ in RPMI-1640 medium with $10 \%$ FBS. We isolated the miRNAs to detect miRNA expression.

RNA isolation and qRT-PCR for miRNA detection. miRNAs were isolated from the cells by using a miRNeasy mini kit (Qiagen, Stanford, CA, USA) according to the manufacturer's protocol. RNA quality was evaluated at 260-280 nm OD ratio. Total RNA (2,000 ng) was reverse-transcribed to cDNA by using a miRcute Plus miRNA first-strand cDNA synthesis kit (Tiangen, China). miRNA quantitation was performed using a TaqMan miRNA assay (Applied Biosystems, Carlsbad, CA, USA) in a CFX96 real-time thermal cycler (Bio-Rad). The 
Table I. Clinical features of patient with ESCC.

\begin{tabular}{|c|c|c|c|c|c|c|c|}
\hline No. & Sex & Age & Location $^{\mathrm{a}}$ & $\mathrm{UICC}^{\mathrm{b}}$ & $\mathrm{UICC} \mathrm{N}^{\mathrm{b}}$ & TNM staging & Tumor grade \\
\hline 1 & M & 72 & $\mathrm{Lt}$ & 4 & 3 & IIIC & M \\
\hline 2 & $\mathrm{M}$ & 53 & $\mathrm{Lt}$ & 3 & 0 & IIA & M \\
\hline 3 & $\mathrm{~F}$ & 67 & Mt & 2 & 0 & IIA & M \\
\hline 4 & M & 44 & Mt & 2 & 0 & IIA & $\mathrm{P}$ \\
\hline 5 & $\mathrm{~F}$ & 64 & Mt & 2 & 0 & IIA & $\mathrm{W}$ \\
\hline 6 & $\mathrm{~F}$ & 58 & $\mathrm{Lt}$ & 3 & 1 & IIIA & $\mathrm{M}$ \\
\hline 7 & $\mathrm{~F}$ & 72 & UtMt & 3 & 0 & IIB & W \\
\hline 8 & $\mathrm{~F}$ & 77 & $\mathrm{Lt}$ & 2 & 0 & IIA & $\mathrm{W}$ \\
\hline
\end{tabular}

${ }^{a} U t$, upper thoracic esophagus; Mt, middle thoracic esophagus; $\mathrm{Lt}$, lower thoracic esophagus. ${ }^{\mathrm{b}} \mathrm{UICC}, \mathrm{N}$ and $\mathrm{T}$ stage are described according to UICC (International Union Against Cancer) TNM Classification (6th edition, 2002). ${ }^{\mathrm{W}} \mathrm{W}$, well; M, moderately; P, poorly.

oligonucleotides used in this study are listed in Table II. All of the samples were normalized using U6, and their relative expression levels were calculated using the $2^{-\Delta \Delta \mathrm{CT}}$ method.

Chip data. The gene expression omnibus (GEO) database of NCBI (National Center for Biotechnology Information) is a public database that stores chip data, next generation sequencing data, and other high-throughput experimental data (http://www.ncbi.nlm.nih.gov/geo/). The accession number of the mRNA Chip data is GSE20347, which includes 17 pairs of ESCC tissues and paired normal adjacent tissues. The chip used for mRNA analysis is the Affymetrix Human Genome U133A 2.0 Array (GPL571), which comprises 47,000 transcriptions and variants covering 38,500 human genes with identified functions.

Identification of differentially expressed mRNAs. GSE20347 was used as an input into BRB-array tools (version 4.5.0 Stable Release). The expression data of mRNA were standardized relative to the reference array, and the median array was used as a reference with the following filtration criteria: i) the signal value of a probe should be 1.5 -fold of the median (bidirectional) and the changes should be $\geq 20 \%$ of the total expression value; ii) the missing value should be $\leq 50 \%$; iii) if a number of probes have been used for one gene, only the probe with the highest IQR is retained. Differential analysis was performed using the class comparison tool of BRB-array tools. Differential mRNA was defined as $\mathrm{P}<0.01$, FDR $<0.01$, and the folds of expression change were $\geq 2$ or $\leq 0.5$.

miRNA prediction analysis and interaction network with $m R N A s$. TargetScan 7.0 and microT-CDS were used to identify the predicted miRNA targets. If the number of target genes was too large, we selected the first 600 genes sorted by the cumulative weighted context score in TargetScan and miTG score in microT. After intersecting the predictions with GEO differential mRNAs, we finally obtained the special genes targeted by miRNAs in ESCC. The interaction network between miRNAs and mRNAs was visualized using Cytoscape.

Interaction network and functional annotation of differential miRNAs and mRNAs. Genes were uploaded to Cytoscape
Table II. The oligonucleotides used in this study.

\begin{tabular}{ll}
\hline Name $^{\mathrm{a}}$ & \multicolumn{1}{c}{ Sequence (5'-3') } \\
\hline U6 F & TGC GGG TGC TCG CTT CGG CAG C \\
miR-6125 F & TTA TTG CGG AAG GCG GAG CGG \\
miR-1285-3p F & GTC TGG GCA ACA AAG TGA GAC CT \\
miR-21-5p F & GCG GTA GCT TAT CAG ACT GAT GTT G \\
miR-424-3p F & GCA AAA CGT GAG GCG CTG CTA T \\
miR-4697-5p F & TAG GGG GCG CAG TCA CTG AC \\
miR-5194 F & ATG AGG GGT TTG GAA TGG GAT GG \\
miR-652-5p F & CAA CCC TAG GAG AGG GTG CC \\
miR-29c-5p F & GTG ACC GAT TTC TCC TGG TGT TC \\
miR-30a-3p F & GCT TTC AGT CGG ATG TTT GCA GC \\
miR-378a-3p F & ACT GGA CTT GGA GTC AGA AGG C \\
miR-378i F & GAC TGG ACT AGG AGT CAG AAG G \\
\hline
\end{tabular}

${ }^{\mathrm{a}} \mathrm{F}$, forward primer.

software for further analysis. The BiNGO plugin (14) in Cytoscape was used to complete GO (Gene Ontology) analysis. Biological process (BP), molecular function (MF), and cellular component (CC) networks were analyzed. The following filtration criteria were considered: i) Benjamini and Hochberg FDR correction for multiple testing correction; ii) significance level $\mathrm{P}<0.05$; lists were sorted by the corrected $\mathrm{P}$-value. The first ten $\mathrm{GO}$ were selected for further clustering. MCODE plugin (15) in Cytoscape was used to find clusters or highly interconnected regions in these three GO networks. DAVID online software (16) was used to perform Kyoto Encyclopedia of Genes and Genomes (KEGG) signaling pathway analysis. Options were set as follows: i) gene count $>2$; ii) display by Benjamini; and iii) $\mathrm{P}<0.05$.

Statistical analysis. Each experiment was conducted at least in triplicate. Data are presented as means \pm standard deviation for each group. One-way ANOVA and Student's t-test in SPSS version 17.0 (Chicago, IL, USA) were carried out. $\mathrm{P}<0.05$ was considered significant. 


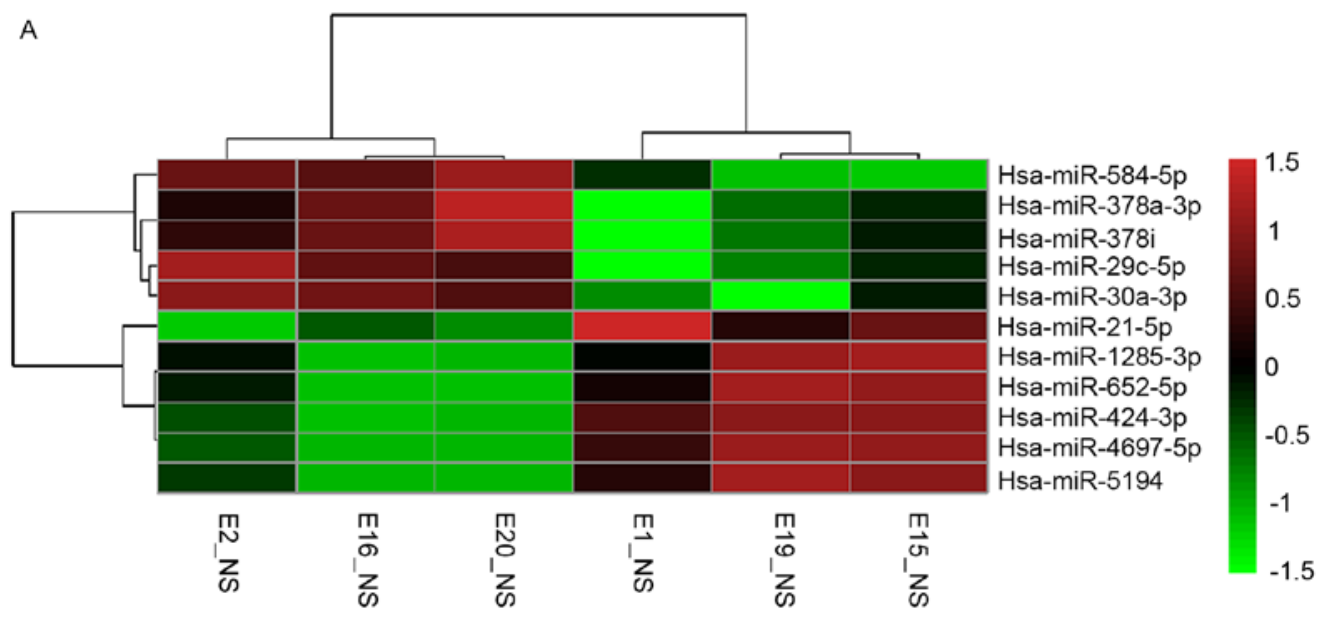

B Tissue state

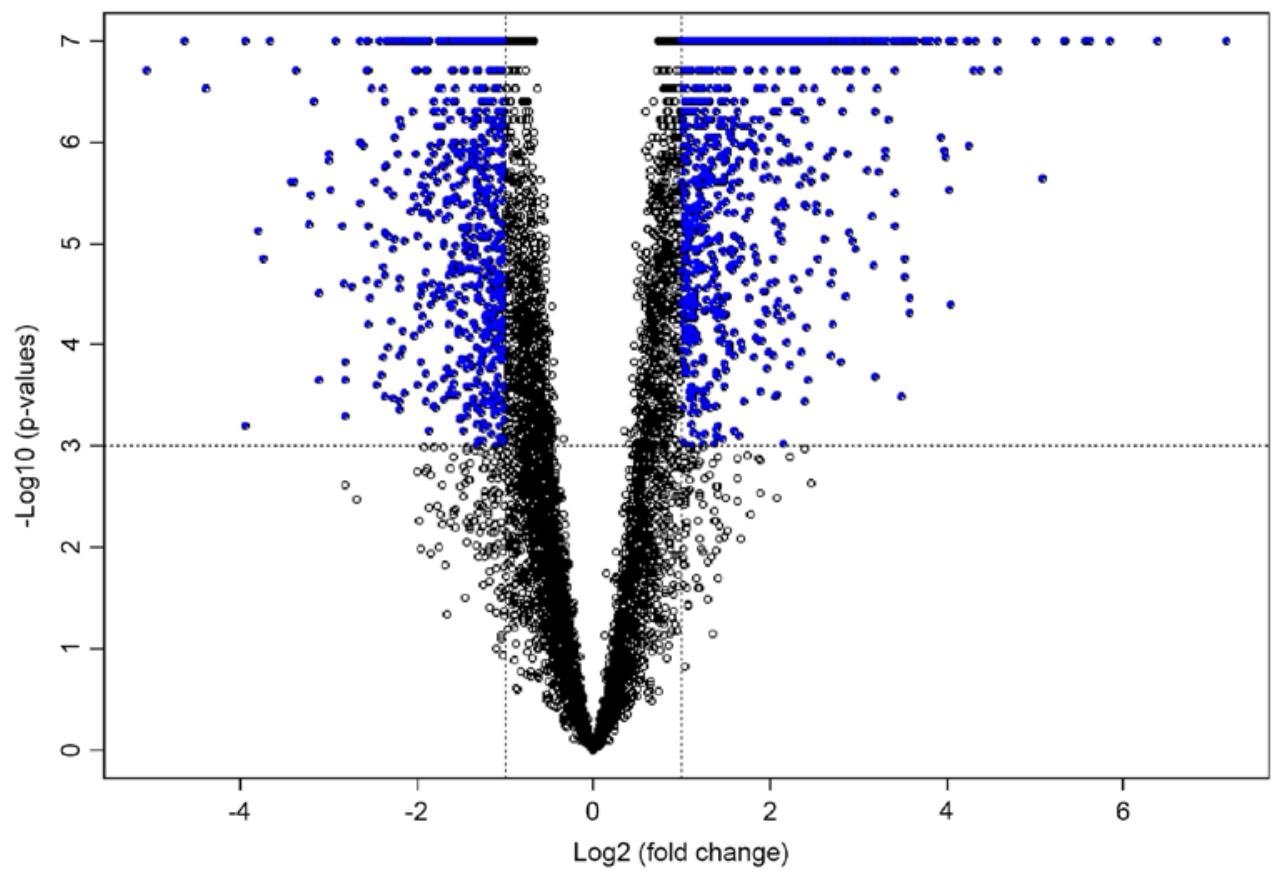

Figure 1. (A) Heatmap of dysregulated miRNAs in ESCC (E15, E19 and E1) compared with paired normal adjacent tissues (E20, E16 and E2). Red indicates upregulation and green denotes downregulation. (B) VolcanoPlot of 1,163 differentially expressed mRNAs by GEO20347 chip data (blue nodes indicate $\mathrm{P}<0.01, \mathrm{FDR}<0.01$, and the folds of expression change $\geq 2$ or $\leq 0.5$ ).

\section{Results}

miRNA expression profile of ESCC. To determine the effect of miRNAs on the development and progression of ESCC in Kazakhs, we initially performed an miRNA microarray analysis (cutoff, $\mathrm{P}<0.05,>2$.0-fold change) with three paired ESCC and adjacent tissues. Our results showed that six miRNAs (miR-1286-3p, miR-21-5p, miR-424-3p, miR-4697-5p, miR-5194, and miR-652-5p) were significantly overexpressed and five miRNAs (miR-29c-5p, miR-30a-3p, miR-378a-3p, miR-378i, and miR-584-5p) were weakly expressed in ESCC tissues compared with normal esophageal tissues (Fig. 1A).

ECA109T1 exhibits stronger invasiveness and motility than ECA109 cells. After completing one selection cycle for highly invading cells, we obtained ECA109T1 with high invasiveness. A Matrigel Transwell invasion assay was conducted to detect the differences in invasiveness between ECA109T1 and ECA109. Cells of ECA109T1 group invaded at a rate of $78 \pm 9.494$ to the lower chamber, whereas ECA109 group invaded at 27.60 $3.076(\mathrm{P}<0.01, \mathrm{n}=5)$. This finding demonstrated that the invasiveness of ECA109T1 was more significant than that of ECA109 (Fig. 2A), also indicated that this cell model could be used to select key miRNAs that participate in tumor metastasis.

NDP-treated ECA109 cell line suppresses proliferation. The cytotoxicity assay via CCK- 8 was carried out to detect the inhibition rate of ECA109 and ECA109 proliferation by $64 \mathrm{mg} / \mathrm{l}$ of NDP at 24, 48, and $72 \mathrm{~h}$. Our results revealed that the inhibition rate was increased with time $(\mathrm{P}<0.05)$ (Fig. 2B). Thus, NDP could suppress cell proliferation and elicit an 


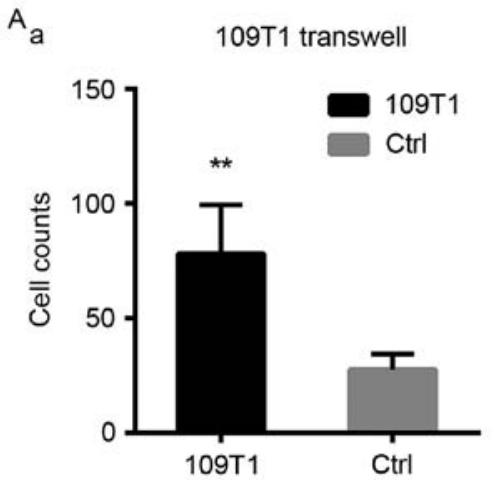

b

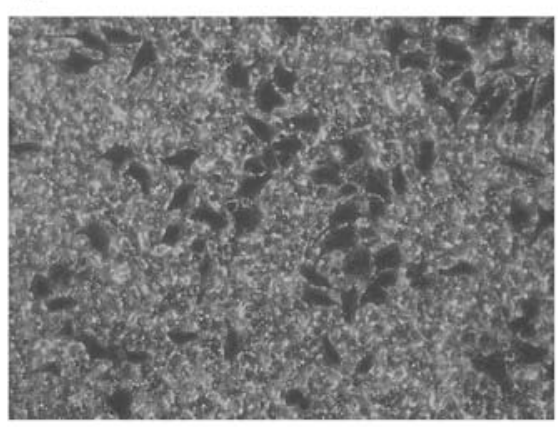

Ctrl
B

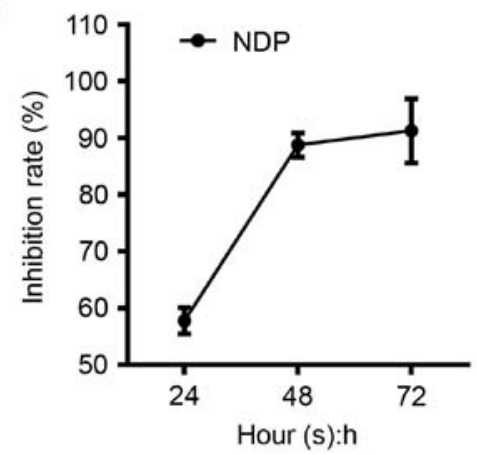

C

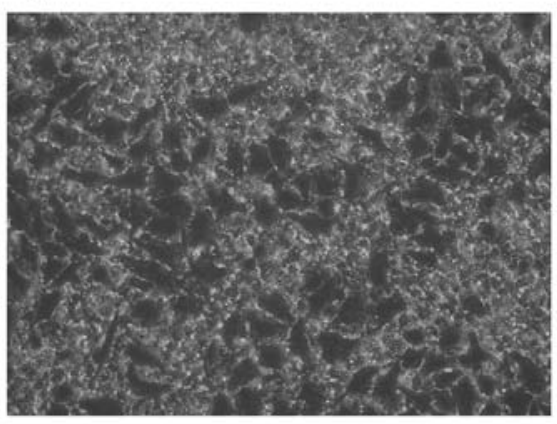

109T1

Figure 2. Characterization assay in ECA109 and sublines (ECA109T1 and ECA109 treated with 64 mg/1 NDP). (A) In vitro invasion activity of ECA109 and invasion cell model ECA109T1 (original magnification, $\mathrm{x} 100$ ). ECA109T1 had higher invasiveness. ${ }^{* *} \mathrm{P}<0.01$. (B) CCK-8 cytotoxicity assay in 64 mg/1 NDP treated EC109 at 24, 48 and $72 \mathrm{~h}(\mathrm{P}<0.05$ compared with the control group). We administered $64 \mathrm{mg} / 1 \mathrm{NDP}$ for $48 \mathrm{~h}$ to 35 -mm culture dish to construct the cytotoxicity cell model.

antiproliferative effect. The expected results could not be achieved because of the state of NDP-treated ECA109 at $72 \mathrm{~h}$. ECA109 treated with $64 \mathrm{mg} / \mathrm{l}$ NDP for $48 \mathrm{~h}$ was used to construct the cytotoxicity cell model and further subjected to RT-qPCR analysis.

$R T-q P C R$ revealed miRNAs with consistent expression. To determine the miRNAs that participate in ESCC cell invasion and proliferation, we performed RT-qPCR detection and quantitated each miRNA from miRNA profiling within the two cell models (Fig. 3). After the qRT-PCR results were compared with the microarray results, an upregulated gene was released by the microarray, and miR-652-5p and miR-21-5p were overexpressed in ECA109T1 and suppressed in NDP-treated ECA109 cells. Therefore, miR-652-5p and miR-21-5p were consistently expressed in the miRNA microarray and cell models of ESCC. Moreover, these two miRNAs may be critical in ESCC occurrence and development. These miRNAs were further used for bioinformatics analyses.

Differentially expressed $m R N A$ screening and interaction network with miRNAs. A total of 650 target mRNAs were present in miR-21-5p and 713 targets were found in miR-652-5p. The mRNA (GSE20347) data were downloaded and integrated in the BRB-array tools for standardization and filtration. A total of 6,572 probes were selected for further analysis (Fig. 1B). The class comparison tool of BRB-array tools was used to analyze chip data. A total of 1,163 mRNAs exhibited differential expression $(\mathrm{P}<0.01$, FDR $<0.01$, and the expression fold-change of $\geq 2$ or $\leq 0.5$ ). Of these mRNAs,

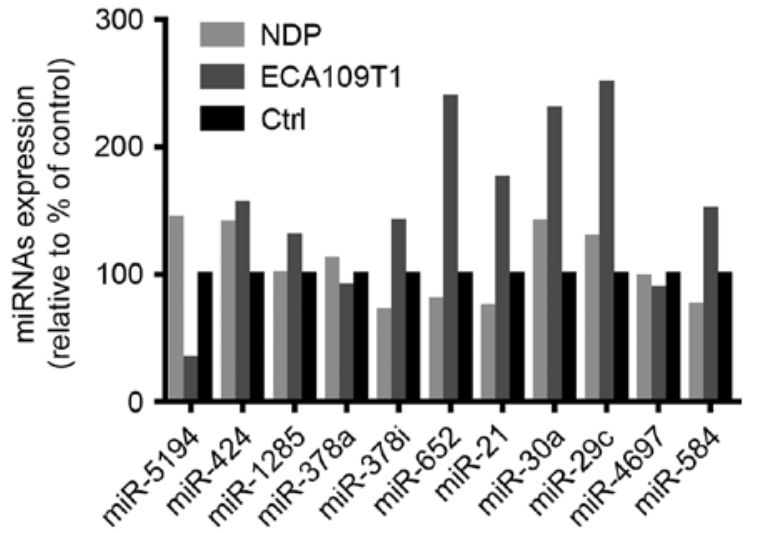

Figure 3. RT-qPCR results of miRNA expression in invasion and cytotoxicity cell models. These results were normalized to U6 expression. miR-21-5p and miR-652-5p were consistently expressed in cell models and miRNA microarray.

640 were upregulated and 521 were downregulated. The intersected genes of miR-21-5p were 59 (35 upregulated and 24 downregulated) and those of miR-652-5p were 38 (23 upregulated and 15 downregulated). The interaction network of miRNAs and intersected genes was visualized by using Cytoscape (Fig. 4).

Network analysis of GO, KEGG, and PPI interactions. GO analysis was performed using the BiNGO plugin in Cytoscape. The first $10 \mathrm{BP}$ and MF were arranged according to the P-value of GO and were chosen for further MCODE 


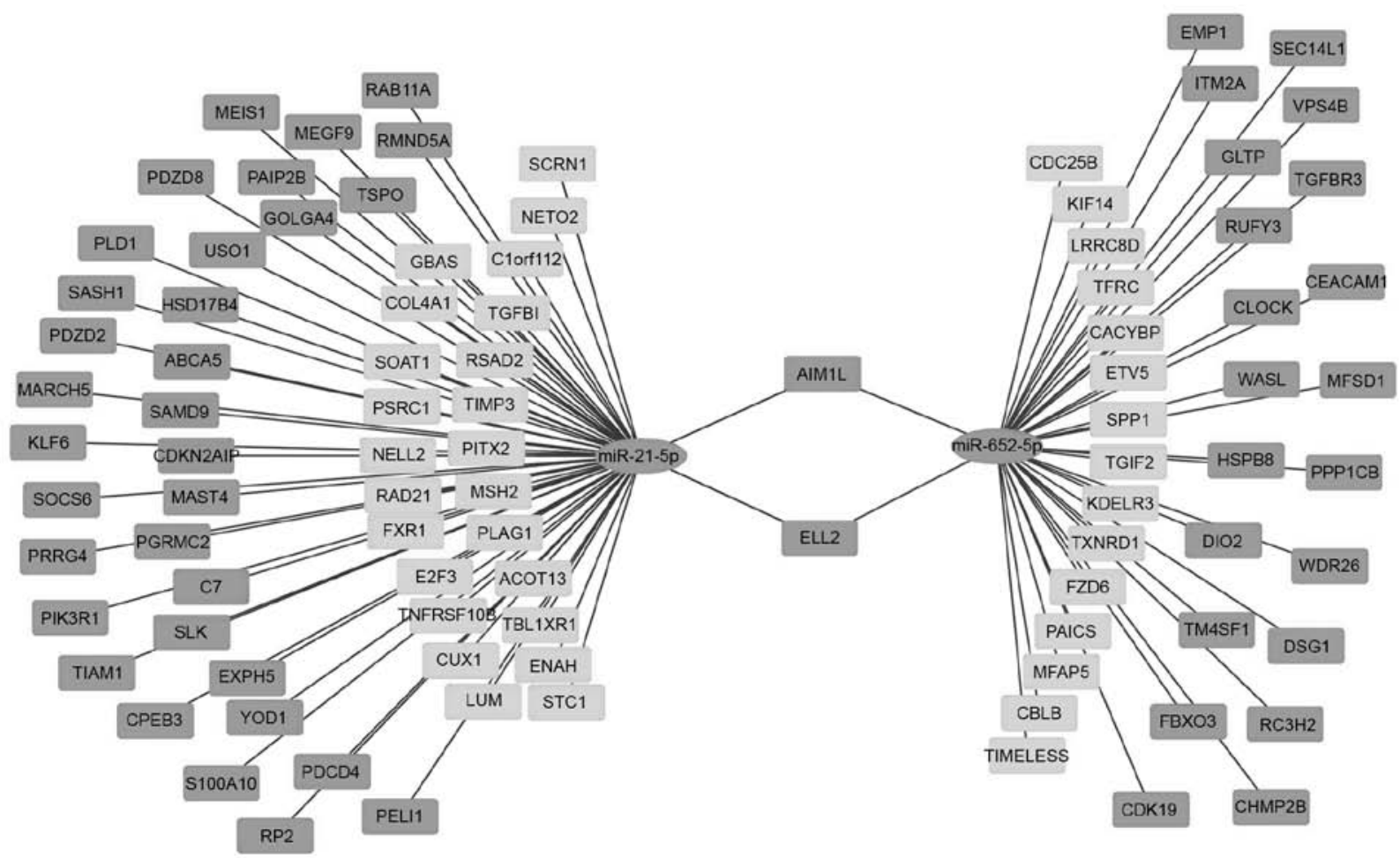

Figure 4. miRNA/mRNA interactive network. miR-21-5p, miR-652-5p, and their potential target genes selected by ESCC microarray revealed by Cytoscape. Ellipse represents miRNAs and rectangle represents target mRNAs. Dark gray indicates upregulation and light gray denotes downregulation.

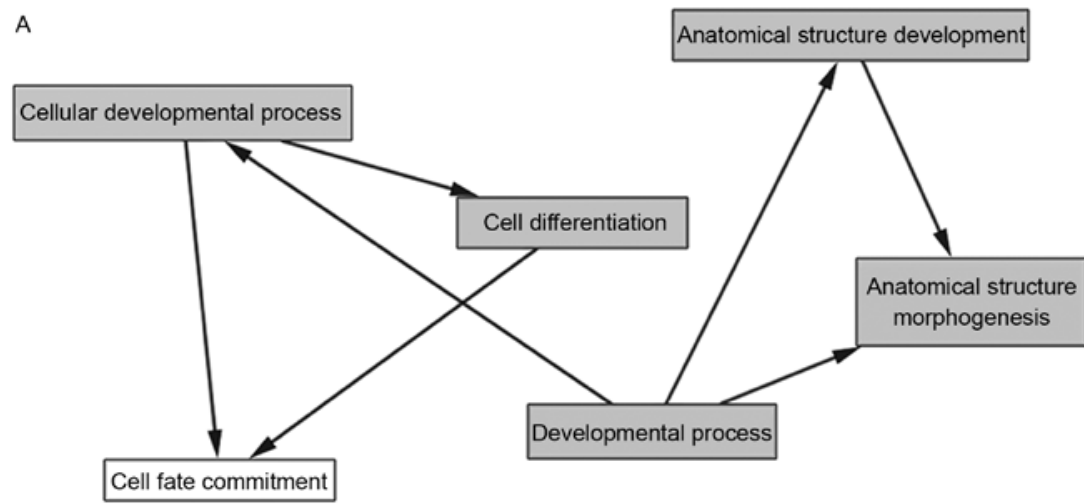

B

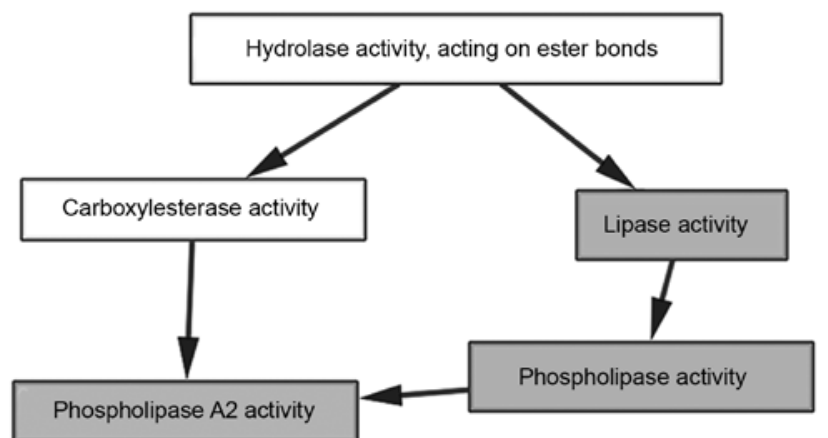

Figure 5. Gene function annotation by Cytoscape plugins. (A) BiNGO and MCODE analysis of biological process by dysregulated mRNAs of the miRNA/ mRNA interactive network. (B) BiNGO and MCODE analysis of molecular function by dysregulated mRNAs of the miRNA/mRNA interactive network. Gray indicates nodes with $\mathrm{P}<0.05$.

clustering analysis. No nodes were displayed in $\mathrm{CC}$ if P-value was $<0.05$. These results indicated that $8 \mathrm{GO}$ and 5 related genes were essential for ESCC GO. BP was as follows: i) cellular developmental process, ii) developmental process, iii) cell differentiation, iv) anatomical structure development, and v) anatomical structure morphogenesis (Fig. 5A). MF was 
Table III. Key genes in GO clusters.

\begin{tabular}{lll}
\hline miRNA & $\mathrm{Up}^{\mathrm{a}}$ & \multicolumn{1}{c}{ Down $^{\mathrm{a}}$} \\
\hline miR-21-5p & PLD1 & MSH2, STC1 \\
miR-652-5p & DSG1 & N/A
\end{tabular}

${ }^{a} U p$, overexpression genes; Down, downregulated genes. ${ }^{b} \mathrm{~N} / \mathrm{A}$, no targeted key genes.

as follows: i) phospholipase activity, ii) lipase activity, and iii) phospholipase A2 activity (Fig. 5B). The mRNAs targeted by dysregulated miRNAs are listed in Table III. DAVID KEGG pathway analysis showed three pathway records: i) endocytosis, ii) pathways in cancer, and iii) actin cytoskeleton regulation (Table IV).

\section{Discussion}

This study aimed to analyze the molecular mechanisms of invasion and proliferation by considering miRNA expression profiles, cell models, and bioinformatics. We obtained 11 differential miRNAs from the microarray profiles of Kazakh ethnicity ESCC and normal adjacent tissues. The ESCC cell line subpopulation with a high invasive ability and cytotoxicity cell model treated with NDP was constructed. However, further studies should be performed to investigate whether these models can decrease the number of miRNAs. Combined with the profile, miR-21-3p and miR-652-3p were further analyzed. Bioinformatics was applied to observe the molecular functions of miRNAs macroscopically. The target genes of miRNAs were obtained via the prediction databases and combined with the GEO data of the ESCC mRNA via BRB-array tools. Cytoscape and its plugin contributed to GO classification and enrichment analysis. In these two miRNA targets, PLD1, MSH2, STC1, and DSG1 may be implicated in cancer occurrence and development.

ESCC in Northwestern China, especially in Xinjiang where the high-risk Kazakh population resides, has a high motility (17). First, some behavioral and environmental risk factors play important roles in ESCC development among Kazakhs (2). After 10 years of national integration, environmental and behavioral factors have decreased. Thus, gene susceptibility and polymorphism are involved (18). Second, Xinjiang is considered an important area for ESCC investigation because of the presence of multiracial society and high incidence. To determine special miRNAs with racial specificity, we collected three paired Kazakh ESCC and adjacent tissue samples for further detection. The collection of tissue from Kazakhs is challenging because of religion, concept, and other factors. However, we will consider additional factors and increase the sample size in our future studies. Our results revealed that miR-21-3p, miR-652-3p, and their potential targets may be involved in ESCC invasion and proliferation.

Bioinformatics approaches are the main patterns used to analyze microarray profiling results and predict miRNA functions $(19,20)$. However, bioinformatics depends on experimental databases, and we found that this approach may easily eliminate miRNAs that have been discovered with few experimental bases. In our experiment, the overbalance in the data size of bioinformatics was related to the databases between miRNAs discovered earlier and later. False-negative results may increase when one method is used. Therefore, two different cell models were constructed in this study. ECA109 is a well-differentiated ESCC cell line from the middle esophagus of a Chinese patient. ECA109T1 is significantly more invasive than its parent cells. Moreover, the mice injected with invasive subpopulation selected by Transwell chambers had faster tumor growth and metastasis (12). NDP is a new platinum derivative selected from a series of platinum analogs based on its pronounced preclinical antitumor activity against various solid tumors with low nephrotoxicity (21). It can be combined with paclitaxel and other chemotherapeutic agents to improve treatment effects and reduce adverse effects. In vitro, NDP affects ESCC proliferation and apoptosis (13). We used NDP to construct a cytotoxicity cell model and investigate its therapeutic effects. However, further studies should determine whether the combination of different chemotherapeutic agents differentiates microenvironment or molecular mechanisms.

qRT-PCR revealed the genes differentially expressed in two cell models. After qRT-PCR findings were matched with microarray results, miR-21-5p and miR-652-5p showed that they may be important in ESCC invasion and proliferation. miR-21 is an oncogene in various diseases, and its overexpression in tissues and serum is associated with poor prognosis (22). Although miR-21 is considered a potential therapeutic target and biomarker of ESCC $(23,24)$, the relationship between miR-652-5p and ESCC has yet to be elucidated. As such, this parameter will be our next focus. Bioinformatics analysis also demonstrated that dysregulated miRNAs and their target

Table IV. KEGG annotation.

\begin{tabular}{lll}
\hline Term & P-value $^{\mathrm{a}}$ & \multicolumn{1}{c}{ Genes } \\
\hline Endocytosis & 0.005 & PLD1, CBLB, TFRC, VPS4B, RAB11A, CHMP2B \\
Pathways in cancer & 0.015 & E2F3, PLD1, CBLB, COL4A1, MSH2, PIK3R1, FZD6 \\
Regulation of actin cytoskeleton & 0.044 & ENAH, TIAM1, WASL, PPP1CB, PIK3R1
\end{tabular}

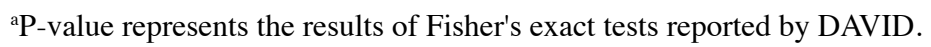


genes (PLD1, MSH2, STC1, and DSG1) were mostly involved in cell differentiation, anatomical structure (BP), and lipase and phospholipase activities (MF). Phospholipase D1 (PLD1) is a phospholipid-metabolizing enzyme whose overexpression promotes cell growth and proliferation by activating Akt (25). Zhang et al revealed that the downregulated PLD1 inhibits the proliferation of gastric carcinoma cells and this enzyme is targeted by miR-638 (26). Human MutS homolog 2 (MSH2) encodes the DNA repair protein. In a clinical survival analysis, MSH2 expression is related to metastasis and tumor shape (27) and recognized as a target by miR-21 (28). The reduced desmoglein-1 (DSG1) expression in cervical tumors is associated with survival and prognosis (29). The association between miRNAs and these four target genes should be determined in vivo and in vitro in future molecular biological studies. Fatty acids are synthetized rapidly in growing tumors (30). In clinical research, the pharmacological control of Fas/FasL signaling may improve the therapeutic efficacy and outcome in ESCC patients receiving preoperative chemoradiotherapy (31). Lipase and phospholipase activation may suppress ESCC development. These target genes likely participate in three pathways, namely, endocytosis, cancer pathways, actin cytoskeleton regulation, which are mostly related to cancer. Furthermore, miR-21-5p and miR-652-5p may perform vital functions in ESCC.

Finally, we successfully selected differentially expressed miRNA and their potential target genes through cell modeling and bioinformatics analysis. Our results indicated that these miRNAs and targets may be used for future therapeutic investigations. These selection processes may improve the efficiency of microarray selection. In the future, miRNAs and their targets should be verified, multiple drugs should be combined, and other supplements should be administered to improve the conclusion of this study.

\section{Acknowledgements}

The authors would like to acknowledge the help from First Hospital Affiliated to Medical College of Shihezi University. This study was supported in part by doctor/scientific grant 2014BB019 from Xinjiang Production and Construction Corps and NFSC $(81260301,81360358,81460362)$ from the National Science Foundation of China.

\section{References}

1. Torre LA, Bray F, Siegel RL, Ferlay J, Lortet-Tieulent J and Jemal A: Global cancer statistics, 2012. CA Cancer J Clin 65 87-108, 2015.

2. Zheng S, Vuitton L, Sheyhidin I, Vuitton DA, Zhang Y and Lu X: Northwestern China: a place to learn more on oesophageal cancer. Part one: behavioural and environmental risk factors. Eur J Gastroenterol Hepatol 22: 917-925, 2010.

3. Lee RC, Feinbaum RL and Ambros V: The C. elegans heterochronic gene lin-4 encodes small RNAs with antisense complementarity to lin-14. Cell 75: 843-854, 1993.

4. Tay Y, Zhang J, Thomson AM, Lim B and Rigoutsos I: MicroRNAs to Nanog, Oct4 and Sox 2 coding regions modulate embryonic stem cell differentiation. Nature 455: 1124-1128, 2008.

5. Stark A, Brennecke J, Bushati N, Russell RB and Cohen SM Animal microRNAs confer robustness to gene expression and have a significant impact on 3'UTR evolution. Cell 123: 1133-1146, 2005.
6. Ferracin M, Veronese A and Negrini M: Micromarkers: miRNAs in cancer diagnosis and prognosis. Expert Rev Mol Diagn 10: 297-308, 2010.

7. Bartel DP: MicroRNAs: Genomics, biogenesis, mechanism, and function. Cell 116: 281-297, 2004.

8. Kan T and Meltzer SJ: MicroRNAs in Barrett's esophagus and esophageal adenocarcinoma. Curr Opin Pharmacol 9: 727-732, 2009.

9. Chen Z, Li J, Tian L, Zhou C, Gao Y, Zhou F, Shi S, Feng X, Sun N, Yao R, et al: miRNA expression profile reveals a prognostic signature for esophageal squamous cell carcinoma. Cancer Lett 350: 34-42, 2014.

10. Takeshita N, Hoshino I, Mori M, Akutsu Y, Hanari N, Yoneyama Y, Ikeda N, Isozaki Y, Maruyama T, Akanuma N, et al: Serum microRNA expression profile: miR-1246 as a novel diagnostic and prognostic biomarker for oesophageal squamous cell carcinoma. Br J Cancer 108: 644-652, 2013.

11. Hurst DR, Edmonds MD and Welch DR: Metastamir: The field of metastasis-regulatory microRNA is spreading. Cancer Res 69: 7495-7498, 2009.

12. Chen YK, Chang WS, Wu IC, Li LH, Yang SF, Chen JY, Hsu MC, Chen SH, Wu DC, Lee JM, et al: Molecular characterization of invasive subpopulations from an esophageal squamous cell carcinoma cell line. Anticancer Res 30: 727-736, 2010.

13. Su XY, Yin HT, Li SY, Huang XE, Tan HY, Dai HY and Shi FF: Intervention effects of nedaplatin and cisplatin on proliferation and apoptosis of human tumour cells in vitro. Asian Pac J Cancer Prev 13: 4531-4536, 2012.

14. Maere S, Heymans K and Kuiper M: BiNGO: A Cytoscape plugin to assess overrepresentation of gene ontology categories in biological networks. Bioinformatics 21: 3448-3449, 2005.

15. Bader GD and Hogue CWV: An automated method for finding molecular complexes in large protein interaction networks. BMC Bioinformatics 4: 2, 2003.

16. Huang W, Sherman BT and Lempicki RA: Systematic and integrative analysis of large gene lists using DAVID bioinformatics resources. Nat Protoc 4: 44-57, 2009.

17. Cui X, Zhao Z, Liu D, Guo T, Li S, Hu J, Liu C, Yang L, Cao Y, Jiang J, et al: Inactivation of miR-34a by aberrant CpG methylation in Kazakh patients with esophageal carcinoma. J Exp Clin Cancer Res 33: 20, 2014.

18. Zheng S, Vuitton L, Sheyhidin I, Vuitton DA, Zhang Y and Lu X: Northwestern China: a place to learn more on oesophageal cancer. Part two: gene alterations and polymorphisms. Eur J Gastroenterol Hepatol 23: 1087-1099, 2011.

19. Johnson CH, Ivanisevic J, Benton HP and Siuzdak G: Bioinformatics: The next frontier of metabolomics. Anal Chem 87: 147-156, 2015.

20. Zhao Y, Zhang X, Zhao Y, Kong D, Qin F, Sun J and Dong Y: Identification of potential therapeutic target genes, key miRNAs and mechanisms in acute myeloid leukemia based on bioinformatics analysis: Med Oncol 32: 152, 2015.

21. Kameyama Y, Okazaki N,Nakagawa M, Koshida H, Nakamura M and Gemba M: Nephrotoxicity of a new platinum compound, 254-S, evaluated with rat kidney cortical slices. Toxicol Lett 52: 15-24, 1990.

22. Fu W, Pang L, Chen Y, Yang L, Zhu J and Wei Y: The microRNAs as prognostic biomarkers for survival in esophageal cancer: A meta-analysis. Scientific World J 2014: 523979, 2014.

23. Liu T, Liu Q, Zheng S, Gao X, Lu M, Yang C, Dai F, Sheyhidin I and Lu X : MicroRNA-21 promotes cell growth and migration by targeting programmed cell death 4 gene in Kazakh's esophageal squamous cell carcinoma. Dis Markers 2014: 232837, 2014.

24. Komatsu S, Ichikawa D, Takeshita H, Konishi H, Nagata H, Hirajima S, Kawaguchi T, Arita T, Shiozaki A, Fujiwara H, et al: Prognostic impact of circulating miR-21 and miR-375 in plasma of patients with esophageal squamous cell carcinoma. Expert Opin Biol Ther 12 (Suppl 1): S53-S59, 2012.

25. Gozgit JM, Pentecost BT, Marconi SA, Ricketts-Loriaux RSJ, Otis CN and Arcaro KF: PLD1 is overexpressed in an ER-negative MCF-7 cell line variant and a subset of phospho-Akt-negative breast carcinomas. Br J Cancer 97: 809-817, 2007.

26. Zhang J, Bian Z, Zhou J, Song M, Liu Z, Feng Y, Zhe L, Zhang B, Yin Y and Huang Z: MicroRNA-638 inhibits cell proliferation by targeting phospholipase D1 in human gastric carcinoma. Protein Cell 6: 680-688, 2015. 
27. Theocharis S, Klijanienko J, Giaginis C, Rodriguez J, Jouffroy T, Girod A, Point D, Tsourouflis G and Sastre-Garau X: Expression of DNA repair proteins, MSH2, MLH1 and MGMT in mobile tongue squamous cell carcinoma: associations with clinicopathological parameters and patients' survival. J Oral Pathol Med 40: 218-226, 2011.

28. Cheng JY, Nishi H, Yonekawa C, Takara $\mathrm{K}$ and Isaka $\mathrm{K}$ : MicroRNA-21 increases the resistance to CDDP in HeLa cervical carcinoma cells by targeting PDCD4 and MSH2. FASEB J 27: (Suppl) lb576, 2013.

29. Myklebust MP, Fluge $\varnothing$, Immervoll H, Skarstein A, Balteskard L, Bruland O and Dahl O: Expression of DSG1 and DSC1 are prognostic markers in anal carcinoma patients. Br J Cancer 106 756-762, 2012.
30. Medes G, Thomas A and Weinhouse S: Metabolism of neoplastic tissue. IV. A study of lipid synthesis in neoplastic tissue slices in vitro. Cancer Res 13: 27-29, 1953.

31. Saigusa S, Tanaka K, Ohi M, Toiyama Y, Yasuda H, Kitajima T, Okugawa Y, Inoue Y, Mohri Y and Kusunoki M: Clinical implications of Fas/Fas ligand expression in patients with esophageal squamous cell carcinoma following neoadjuvant chemoradiotherapy. Mol Clin Oncol 3: 151-156, 2015. 